\title{
Research on Concentrated-model of Transmission Network Management System based on Cloud
}

\author{
Huiping Meng ${ }^{1, a}$, Zhen Luo ${ }^{1, b}$, Lei Sheng ${ }^{1, c}$,Yongqiang Guo ${ }^{1, d}$ \\ ${ }^{1}$ Information \& Telecommunication Co. of State Grid Henan Electric Power Company, \\ Zhengzhou 450052 China \\ aiemengping@126.com, b15617911008@126.com, ${ }^{\mathrm{c}}$ woyaogre@163.com, dgyq@ha.com.cn
}

Keywords: province-centralized; hierarchical province-centralized mode; Network Manager System; Cloud

\begin{abstract}
The electric power transmission network management is changing from decentralized to province-centralized mode to adapt to the development trend of power grid. For the current hierarchical management situation of transmission system, the paper mainly studies the centralized mode. Considering the difficulty of transform, security, the demand of bandwidth and augmentability factors, the paper proposes a hierarchical province-centralized mode based on Resource pool and states its architecture, realization methods and deployment mode of Network Manager System in Cloud, realizing the unified management, configuration and data sharing of the whole province. Deploying the network management system in the Clout is taking full use of the resource sharing, efficient management and on-demand resource of the Cloud to realize the intensification, promoting the scheduling intensive management level of Information and transmission.
\end{abstract}

\section{Introduction}

For a long time, the layered, graded, zoned management mode of electric power communication networks makes the network management system built and maintained by the way of multi-level scheduling mechanism ${ }^{[1]}$. In order to fulfill the development trend of power grid, power transmission network management should be developed from the provinces and cities separated pattern to the province-centralized mode to realize unified management, unified configuration and sharing of the communication network data within province and achieve the purpose of concentrate and highly active management, reasonable resource-configuration and optimizing the structure of the network resources ${ }^{[2]}$.

Deploying the communication network management centrally is an effective way to enhance the level of intensive management, which, whereas, will cause the load that systems deal with increase sharply $^{[3]}$. However, Cloud computing technology integrates resources into form Cloud in order to get higher utilization of computing and storage, having a strong computing power and storage capacity. With the development of Cloud construction, deploying communication network management in the pool can improve data reliability and security. This paper puts forward a hierarchical province-centralized mode based on Cloud by researching on concentrated-model of Transmission Network Management, and describes the model in detail.

\section{Discussed Problems}

Power system communication network is the important technology support and infrastructure platform of the grid. As an important guarantee of power grid security, stability and economic running, communication network management system is the important technology means of the communication network management and maintenance. 


\section{Communication network management system}

At present, the general transmission network management system is divided into Element Management System(EMS), Subnet Management System(SNMS) and Network Management (NMS) in terms of the management level ${ }^{[4]}$. EMS is based on the device manager and realizes the management of safety, configuration, performance and alarm for specific equipment; SNMS can divide the network elements into various subnets, not only manage the elements equipments but also the topological relation between them. NMS is a higher level than the SNMS and manage multiple SNMS systems at the same time through network. It configures the router for each business level, and also shows the network elements' circumstances of each time slot and the business relationships at all levels in details. The relationship of different level network management system is showed as the figure 1.

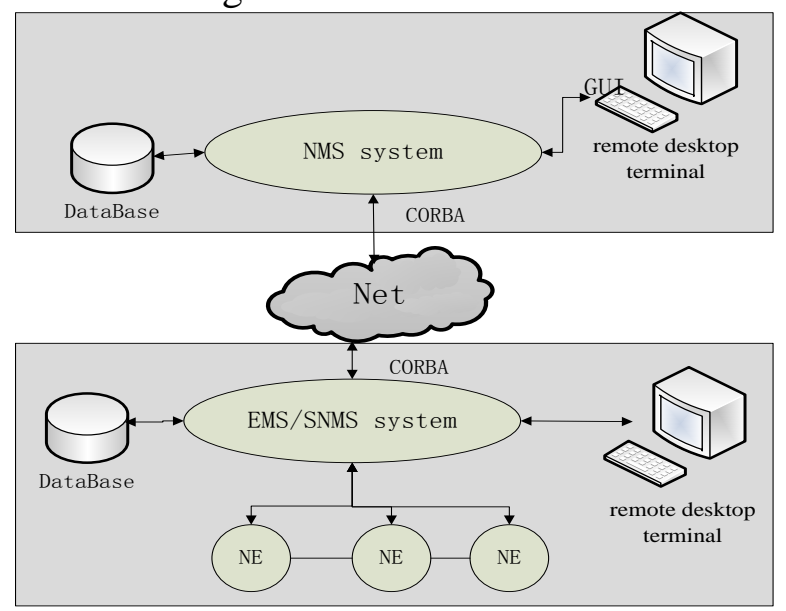

Figure 1: relationship of different level network management system

As the figure 1, EMS or SNMS system connects with network elements through the communication channel, and manage each of Net-Element (NE) directly on equipment level and NE level. Besides, SNMS can also manage relationship of subnet network elements. NMS links with EMS or SNMS system through the network and form a larger network with the various subnets to achieve monitoring and management the entire network equipment with lower interaction through the north Interface, including surveillance network performance, establishing network connectivity, maintaining and tearing down network and locating fault, scheduling, protecting network and User's business, and tracking service quality, etc.. Users can also log in to the NMS system to manage the whole transmission network through the NMS remote desktop terminal GUI(Graphics User Interface).

\section{The difficulties of employing network management centrally}

At present, most of the network management hierarchically construct and operate according to the level of scheduling and manage different levels of network independently. It is an important means to employ the transmission network management systems centrally in provincial capital ${ }^{[5]}$, in which main and standby SNMS systems are built in the communication equipment room of provincial electric company to control data and management of the whole communication network intensively through manage all sub provincial network elements. However, there exist some problems as following:

1) Big difficulties to reconstruct the existing network. It is quite difficult and has a big effect on the existing network, which need to cancel or reset the sub provincial MS and built new SNMS in provincial control centre.

2) Poor security. Since the SNMS systems are just built in the provincial company so that the reliability of the provincial company determine the security of the entire network and any fault of the provincial company system will affect the entire provincial network operation. 
3) Huge bandwidth. For the SNMS in provincial capital needing to connect with the gateway and NE(Network Elements) in sub cities and link with operating terminal in cities and counties, it will need more bandwidth between capital and cities.

4) Weak augmentability. The subject of the network construction and administration is in provincial company so that its flexibility and augmentability are weak.

To sum up, we should make further analysis on the network manage systems, and formulate a province-centralized mode program of Transmission Network based on Cloud according to the feature of electric power.

\section{Hierarchical Provincial-centralized Mode}

Aiming at the peculiarity of the power transmission Network Management, the mode of hierarchical provincial-centralized based on the Cloud reconstructs the existing EMS or SNMS systems and deploys NMS in province company after analyzing factors such as difficulty of reconstruct the existing transformation network, safety, bandwidth consumption, the flexibility and resource intensive and considering the require of the capability of servicer, so that communication networks can be centralized monitored and managed within province.

\section{Mode description.}

Hierarchical provincial-centralized mode means that building main and standby NMS in provincial electric company, and linking with the EMS or SNMS systems of city-region in parallel by LAN (Local Area Network), so that the network management systems are separated from each other in cities and counties can be integrated to realize data concentrated and management centralized.

By managing EMS and SNMS of sub city, the NMS can centralize configure any end-to-end circuit/route and gather NE information and fault information, as well as the security management of the whole network management system, preventing unauthorized operator accessing net resource and net management and taking protective measures for key parts. The overall structure of hierarchical capital concentrated-model is shown in Figure 2:

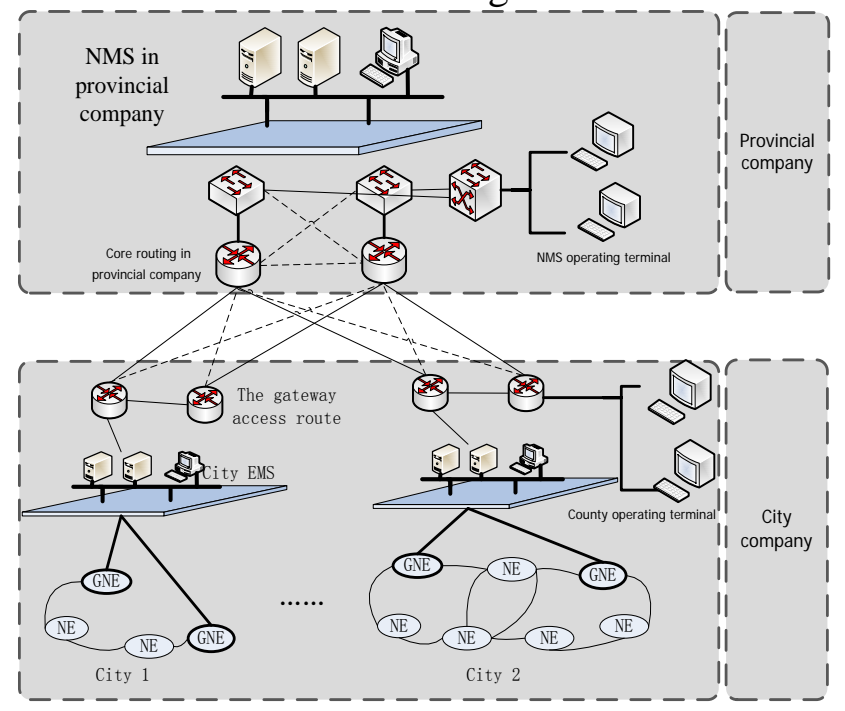

Figure 2: The overall structure of hierarchical capital concentrated-model based on Cloud.

Hierarchical provincial-centralized mode as shown in Figure 2 has fully considered the current situation of the transmission of present power grid. It just needs to deploy NMS in Cloud resource pool of the provincial company, and make use of the hierarchical management model of NMS. The county company network merge into the city one, and the existing network of the city-region company won't to be changed. Besides, county company should relocate and reuse the using service equipment to lower the difficulty of reconstructing the existing network.

Network management system in city company particular emphasis on operating functions such as allocation, breakdown, performance, topology, security; and that in provincial company particular 
emphasis on management functions such as monitoring the whole net resource, statistics and report forms management, as well as the relative performance analysis between fault and performance.

\section{Mode net-setting.}

1) Connection of Network management systems in province and cities: NMS connect with EMS or SNMS through the interface of CORBA. Application of NMS gain and storage data in database through CORBA after being processed, providing the EMS topology managing, allocation managing, fault managing, performance managing, security managing for NMS. The interface design as shown in Figure 3:

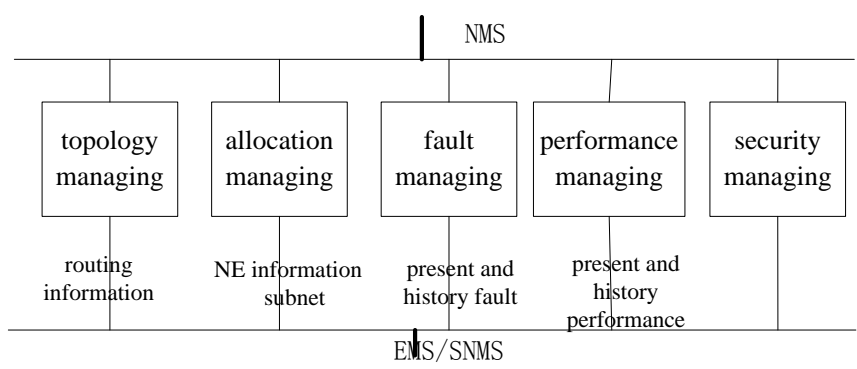

Figure 3: The interface design of CORBA

2) Routing connection: As shown in Figure 2, NMS connect with EMS or SNMS through network. Deploy two convergence routers in the network management centre. Each of them connects through the Ge links. Subnet in each city should have at least one GNE (gateway network element) and one backup GNE. Network equipment will communicate with the management systems in the manner of $1+1$ link backup to realize the connection during cities SNMS and capital NMS. Firstly, NMS communicate with city GNE via TCP/IP, and then NE communicate with nonGNE by ECC, which realizes communication between NMS and NEs in sub city.

3) Subnet partition: according to the trait of regional of the present network, all managed equipment in each local company forms a subnet, and each subnet chooses at least two GNE, one major one backup, to avoid the trouble of more NE lose control caused by single GNE fault. Remote operated terminal is divided into a subnet alone, separated from equipment subnet.

4) Network integration transformation: it will not build network management system itself in county company. Instead, it remove and merge management servers to the city-region company, then transform the server and terminal operator in county company into the remote terminal operator of city-region company.

5) Terminal operator of NMS in provincial company: NMS provides a visual operating interface with GUI. Terminal operators connect with main and standby server of NMS by VLAN cable. Manager in provincial company could check management information and initiate operate request by GUI.

6) Permissions grading: set permission for different levels and limit the remote operating permission strictly. According to the unified centralized management, it defined addressable target, operating control, addressable module strictly to gain different management functions by providing different level safety control measures.

7) IP and ID setting: unify planning and mapping management data of the whole net to avoid conflict of IP and ID. The capacity expansion of sub city companies will not affect the provincial company network, which makes the augmentability and flexibility better.

\section{Deployment of NMS in the Cloud pool}

Cloud resource pool will change traditional from solidified to flexible, elastic and high available; from traditional resources supply mode in which estimating the size of structure according to the maximum load to the mode that resource sharing and on-demand, automatic assembled and dynamic scheduled $^{[6]}$. With the construction of software and hardware Cloud resource pool system 
and the cloud in companies, deploying the network management systems into the resource pool will improve the efficiency of resource utilization and realize resource intensive management.

\section{Deploying the NMS into the Cloud.}

The deployment of NMS network management system needs to consider application and the database program. The main characteristics of the Cloud resource pool are virtualization of data and management automation, though splitting host resources into multiple independent virtual machines and dispatching automatically through virtualization technology as to improve the degree of refinement and efficiency of resource use. Figure 4 shows the deployment scheme of NMS network management system in the Cloud.

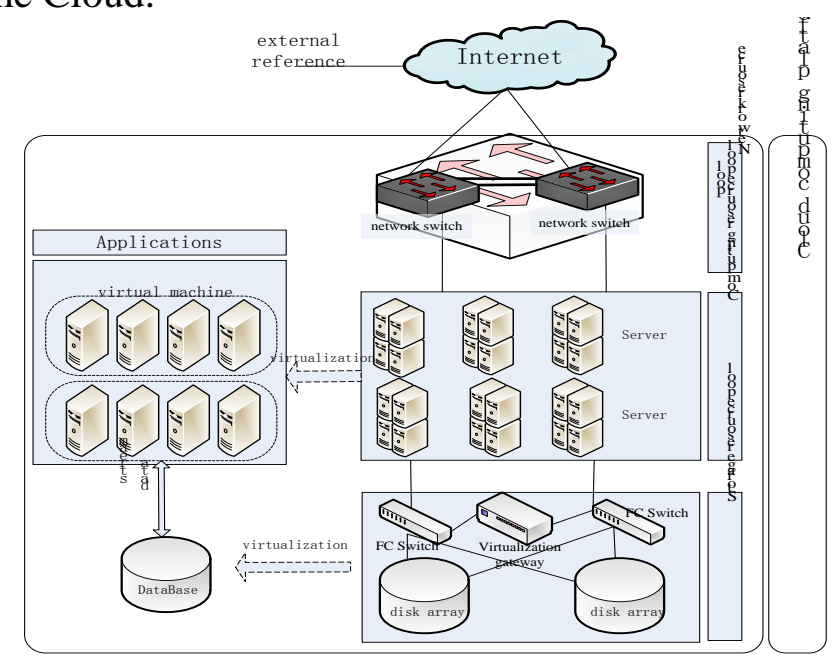

Figure 4: The deployment scheme of NMS in Cloud

As the figure 4, the NMS application service program is deployed in the computing resources layer. The cloud computing platform form more than one virtual server system after integrates the service resources to hosting different program; the communication data during NMS with EMS or SNMS system is stored in the storage pool and the application accesses them through the switches and virtualization gateway. The outside program accesses the NMS application service program and data through the upper network in the Cloud.

Considering the problem of disaster recovery, two sets of NMS network platform as main and standby for each equipment brand should be built and deployed in a different virtual host. Many of the virtual host having same application should be mounted on different physical host to ensure that the application can be used while a single physical machine failure. Each NMS system program should be loaded in more than one virtual machine, and adjust usage of the resource between each virtual machine through load balancing mechanism of the cloud platform

\section{Advantage of deploying the NMS into the Cloud.}

1) High reliability. For the NMS deployed in virtual machine, the application can drift seamlessly in other virtual machines when a single virtual machine went down. On the other hand, applications are isolated from physical platform completely, the cloud resource management platform expands and contracts resources automatically according to business pressure to avoid hardware maintenance and fault impacting system operation.

2) Resource intensification. Cloud use virtualization technology to integrate software and hardware resources so that the business platform construct, implement, operate and maintain uniformly to achieve the resource management and usage intensively.

3) Reduce investment outlay. Deploying the NMS in the Cloud uniformly, so there is no need to equip high-performance hardware resources for each system in provincial centralized mode. Province-centralized transmission network management systems can share the main software and 
hardware equipment, network security equipment and equipment room, power supply, air conditioning and other supporting facilities to reduce the investment cost greatly.

\section{Conclusions}

Province-centralized network management systems will achieve the communication network centralized monitoring, which are conducive to the scheduling mode of the province within the scope of inter-district across the network. It is an effective means for information communication scheduling intensification, and has a great influence over the construction, applications and organization of the process flow of the original network management. For the status of power network management and informationalized reform based on cloud computing resources pool, implementing hierarchical provincial-centralized mode deployed in the resource pool is conform to the principle of the power communication network steady development after considering the present network reconstruction difficulty, security, and bandwidth requirements, and augmentability. At the same time, it takes full use of the sharing characteristics of the resource pool to employ the NMS systems in Cloud pool and improves the utilization of hardware resources. On the other hand, the efficient management and its on-demand characteristics ensure the high demand to the server under high load pressure.

\section{References}

[1] Jian Shi, Yujian Chao, Jun Liu. Research on alarm Standardization of Electric power communication network management system [J]. Information and communication technology of the power, 2013, 11(10):72-76.

[2] Zhiyuan Ma, Yushan Tang, Jian Shi. Centralized power communication comprehensive net management system between province and subcity[J]. Power system communication, 2012, 33(2):10-14.

[3] Jiaru Liang, Bingbing Fan. Research on strategy of application system deployment in Cloud [J]. Computer and modernization, 2013, (8):145-150.

[4] Jun Wu. Research on technology of transmission network Management from the network layer [D]. Huazhong University of Science and Technology,2004.

[5] Wei Liu, Sizu Hou. Research on Distribution network monitoring system [J]. Silicon Valley, 2013, (11):156-156.

[6] Chun Hua, Jun Li, Huan Liang. IT service management implementation strategy under the environment of Resource pool [J]. Telecommunications Science, 2012, 28(9):142-147. 